\title{
Validation of the modified digital element approach for simulating ballistic impact against fragment simulating projectile
}

\author{
Ying $\mathrm{Ma}^{1, \mathrm{a}}$, Youqi Wang ${ }^{2}$ and Tiantian $\mathrm{He}^{1}$ \\ ${ }^{1}$ Department of Advanced Manufacture Engineering, Chongqing University of Posts and Telecommunications, Chongqing, P.R. China \\ ${ }^{2}$ Department of Mechanical and Nuclear Engineering, Kansas State University, Manhattan, KS, USA
}

\begin{abstract}
A modified digital element approach (DEA) is applied to simulate fabric perforation process under ballistic impact. The previous version of the DEA is capable of simulating ballistic impact of textile fabric using rigid body spherical and cylindrical projectiles only. Fragment simulating projectile (FSP) and real bullets are not modeled. The subject of this research is to perform ballistic penetration process against projectile of arbitrary shape and validate the modified DEA. A fabric to solid body projectile contact search and contact force calculation algorithm is established. Ballistic impact of textile fabrics against spherical and cylindrical projectiles is performed using the previous DEA and the modified DEA separately. Numerical results are compared to the well published DEA results to investigate the fabric bullet resistant performance.
\end{abstract}

\section{Introduction}

The strength of textile fabrics under projectile impact is of high importance to soldier protection applications. Bullet resistant body armor is designed to protect soldiers against projectile penetration and blunt trauma. Textile armor is evaluated for both penetration resistance by bullets and for impact energy transmitted to the wearer.

As the development of computer power, yarn level approach became popular in 1990s. Explicit finite element commercial simulation tools, such as LS-DYNA, ABAQUS, and AUTODYN[1-4] are used intensively in failure modeling for impact mechanism research. A damage initiation criterion for the material model, based on the fabric or yarn level constitutive relationship, was implemented in the software as subroutine, which embraces yarn failure, matrix damage, and delamination under tensile, compressive, shear, and crush loadings. Although, the use of finite element method in multiscale 3-D woven textile related mechanics research has provided insight to the fabric response under ballistic impact and failure mechanism, fiber level details of fabric microstructure are overlooked.

A near fiber-level digital element approach (DEA) for simulating impact and penetration of textiles against rigid spherical and cylinderical projectile is established by Wang and Miao[5] in 2010. In this micro-approach, each yarn is discretized into several equal length digital fibers. Digital fibers are modeled as digital chain. Digital chain, imitating the physical behavior of an actual fiber, embodied the sub-yarn scale properties of fabrics. Therefore, fiber strength, fiber-to-fiber contact and friction can be modeled[6]. Figure 1 shows three key elements of DEA and its unit-cell micro-geometry of 3-D woven textile generated by Dynamic Fabric Mechanics
Analyzer(DFMA)[7] utilizing dynamic relaxation process with periodic boundary conditions[8]. In 2016, Wang and Miao[9] implemented a Monte Carlo process to assign a unique strength to each element following a bimodal Weibull distribution function to investigate the effect of inter-fiber friction coefficient on fabric ballistic performance. Although, fiber damage propagation, fabric perforation mechanisms and their relations between interfiber friction coefficient are analyzed in detail, ballistic performance against FSP is never discussed at filament level.

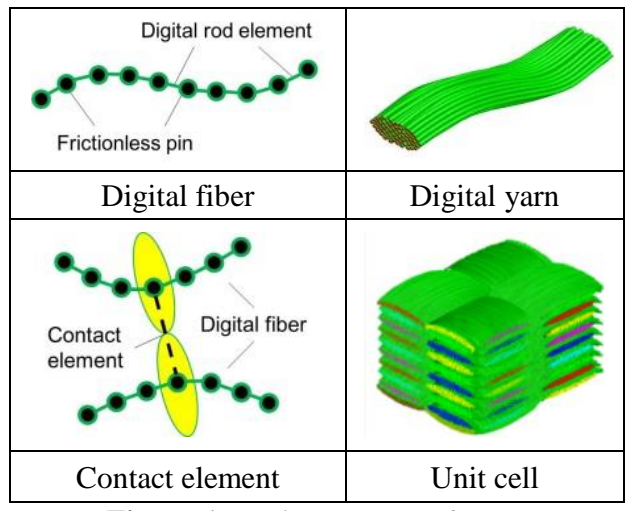

Figure 1. Basic concepts of DEA

In this research, first, the DEA is modified to simulate ballistic impact of textile fabric against solid body projectiles of arbitrary shape. Second, The contact search and contact force calculation between fabric and solid body projectile algorithm is explained in detail. Third, the impact simulation results of solid body spherical and cylinderical projectiles are compared to that of the previous DEA to test and validate the modified DEA capability. Solid conclusions are reached based on numerical results.

* Corresponding author: maying@cqupt.edu.cn 


\section{Ballistic impact to fabric using rigid body projectile of arbitrary shape}

\subsection{Contact search methology}

A node-to-surface contact algorithm is established to model projectile-to-fabric contact. Three steps are taken in preparation for the contact calculation: first, a projectile model is created and discretized with 8-node solid elements using commercial software. Second, elements and nodes belong to the projectile surface are extracted and sorted in structural order. Third, divide the surface elements into sub-triangles and calculate the plane equation and plane normal of each. The nodes in contact with each projectile surface element are determined using point-to-plane distance formula. Figure 2(a) shows an example of meshed FSP and Figure 2(b) demonstrates the contact model. Projectile failure due to impact is not considered at this stage.

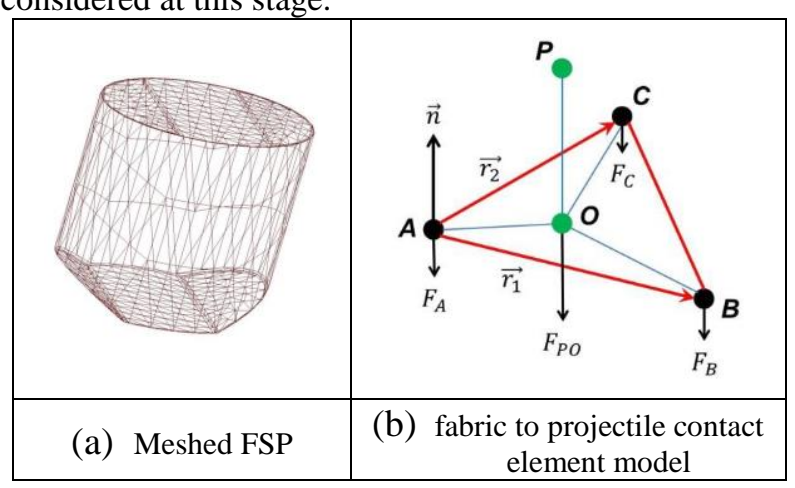

Figure 2. Contact search model

As shown in Figure 2(b), point $P$ is a node on the fabric, triangle element $A B C$ belongs to the projectile surface. When node $P$ encounters triangle $A B C$, a virtual node $O$ is generated to represent the physical point upon which the contact force is applied. Line $P O$ is perpendicular to plane $A B C$ and equals to the distance between node $P$ and plane $A B C$.

To determine wether point $P$ is in contact with surface element $A B C$, two conditions must be filled: 1) the distance between node $P$ and plane $A B C$, calculated by equation(1), must be smaller than the digital fiber radius, 2) point $O$ must be inside triangle $A B C$.

$$
\mathrm{l}_{\mathrm{c}}=\mathrm{n}_{1}\left(\mathrm{x}_{\mathrm{p}}-\mathrm{x}_{\mathrm{A}}\right)+\mathrm{n}_{2}\left(\mathrm{y}_{\mathrm{p}}-\mathrm{y}_{\mathrm{A}}\right)+\mathrm{n}_{3}\left(\mathrm{z}_{\mathrm{p}}-\mathrm{z}_{\mathrm{A}}\right)
$$

where $n_{1}, n_{2}$, and $n_{3}$ are the $x, y$, and $z$ components of the plane normal $\mathbf{n}$. Let vector $\mathbf{r}_{\mathbf{1}}$ be a vector from point $A$ to point $B$ and $\mathbf{r}_{2}$ be a vector from point $A$ to point $C$. The normal vector of triangle $A B C$ can be calculated as $\mathbf{n}=$ $\frac{\mathbf{r}_{1} \times \mathbf{r}_{2}}{\left|\mathbf{r}_{1} \times \mathbf{r}_{2}\right|}$. The direction of $\mathbf{n}$ is determined using the righthand rule and should always point outwards.

If $l_{c}$ is smaller than the digital fiber radius, the location of point $O$ needs to be verified through following procedure: 1) divide triangle $A B C$ into three sub-triangles: $B C O, A C O$, and $A B O$. 2) Calculate the area of each subtriangle $a_{1}, a_{2}, a_{3}$, and the area of triangle $A B C$, denoted as $a$. 3) If $a_{1}+a_{2}+a_{3}=a$, node $O$ is inside triangle $A B C$ and node $P$ and triangle $A B C$ are in contact. Contact force $F_{P O}$ is calculated and distributed proportionally onto node $A$, $B$, and $C$, as such $F_{A}=\frac{a_{1}}{a} F_{P O}, F_{A}=\frac{a_{2}}{a} F_{P O}, F_{A}=\frac{a_{3}}{a} F_{P O}$, and $F_{A}+F_{B}+F_{C}=F_{P O}$. The direction of $F_{A}, F_{B}$, and $F_{C}$ is the same as the direction of $F_{P O}$. Otherwise, node $O$ is outside triangle $A B C$, no contact exists between node $P$ and triangle $A B C$.

\subsection{The modified DEA algorithm}

In a single degree system, the relationship between mass, spring elements and viscous damper can be expressed as:

$$
\left(r_{i}\right)_{e}-\left(k_{i j}\right)_{e} \times\left(u_{i}\right)_{e}-c\left(\ddot{u}_{i}\right)_{e}=\left(m_{i j}\right)_{e} \times\left(\ddot{u}_{i}\right)_{e}
$$

where $\left(r_{i}\right)_{e}$ is the external force of element $e$ applied to node $i,\left(k_{i j}\right)_{e}$ is the element stiffness matrix, and $\left(u_{i}\right)_{e}$ is the relative displacement of element $e$ with respect to its original position. Thus $\left(k_{i j}\right)_{e} \times\left(u_{i}\right)_{e}$ is also known as the internal force. During each time step, element stiffness matrix of each element and the corresponding internal forces of each node are calculated. The nodal forces of each element are assembled to a global force vector. A central difference explicit direction integration algorithm is employed to calculate nodal velocity, acceleration, and displacement of the fabric and projectile.

$$
\begin{gathered}
\left(a_{i}\right)_{n}=\left(F_{i}\right)_{n} / m_{i} \\
\left(v_{i}\right)_{n+1 / 2}=\left(v_{i}\right)_{n-1 / 2}+\left(a_{i}\right)_{n} \Delta t \\
\left(u_{i}\right)_{n+1}=\left(u_{i}\right)_{n}+\left(v_{i}\right)_{n+1 / 2} \Delta t
\end{gathered}
$$

where $i$ is the node number; $n$ is the number of steps; $m$, $F, a, v$, and $u$ represent nodal mass, force, acceleration, velocity, and displacement respectively. In every iteration, a $\Delta t$ smaller than critical time step is used to maintain conditional stability. For finite element (FE) meshed projectile, the internal nodal forces are calculated element by element and distributed onto each node. No global stiffness matrix is generated

\subsection{Validations}

To have the same numerical precondition as the DEA, only spherical and cylindrical projectiles are simulated. A set of 5 and 4 of each projectile type with different mesh density is simulated to evaluate its effect on ballistic performance. The modified DEA in simulating ballistic impact of deformable projectile of arbitrary shape is verified. Simulation details are discussed below.

\subsubsection{Material properties and projectile geometry}

As shown in Figure 3, the fabric used in the simulation is a 1 in. $\times 1$ in. rectangular Kevlar KM2 2-D plain weave generated by DFMA[5,8,9]. Projectile is positioned above the fabric geometric center, with an initial velocity of $50 \mathrm{~m} / \mathrm{s}$ directed towards the fabric. Fixed boundary conditions are applied on all four boundaries. Table 1 and 
Table 2 list the fabric material and projectile properties which are provided by the Army Research Lab.

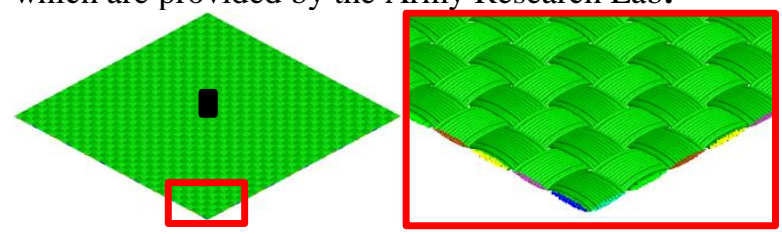

Figure 3. 2-D plain weave fabric

Table 1. Material Properties of Kevlar KM2

\begin{tabular}{ccccc}
\hline $\begin{array}{c}\text { Type } \\
\text { Kevlar }\end{array}$ & E11 $(\mathrm{Pa})$ & E22 $(\mathrm{Pa})$ & Strength $(\mathrm{Pa})$ & $\begin{array}{c}\rho \\
\left(\mathrm{kg} / \mathrm{m}^{3}\right)\end{array}$ \\
KM2 & $8.46 \mathrm{e} 10$ & $1.34 \mathrm{e} 9$ & $3.88 \mathrm{e} 9$ & 1440 \\
\hline
\end{tabular}

Table 2. Projectile Geometry

\begin{tabular}{|l|} 
Projectile type \\
Diameter $(\mathrm{m})$ \\
Mass $(\mathrm{kg})$ \\
Density $\left(\mathrm{kg} / \mathrm{m}^{3}\right)$ \\
$\mathrm{E} 11(\mathrm{~Pa})$ \\
$\mathrm{E} 22(\mathrm{~Pa})$ \\
Height $(\mathrm{m})$ \\
$v$
\end{tabular}

\begin{tabular}{cc|} 
Sphere & RCC \\
$5.56 \mathrm{e}-3$ & $3.40 \mathrm{e}-3$ \\
$6.92 \mathrm{e}-4$ & $2.592 \mathrm{e}-4$ \\
7689 & 7689 \\
$2.09 \mathrm{e} 11$ & $2.09 \mathrm{e} 11$ \\
$2.09 \mathrm{e} 11$ & $2.09 \mathrm{e} 11$ \\
- & 0.0037 \\
0.3 & 0.3
\end{tabular}

\subsubsection{Simulation results and discussion}

The fabric total nodes and projectile mesh density substantially affect computational time spent on calculating contact forces. In general, the higher projectile mesh density, the more accurate the results will be. In order to find the least number of projectile total nodes which produces smallest discrepancy comparing to the well-published results from the previous DEA, projectiles of varies mesh density are simulated, examples are shown in Figure 4.

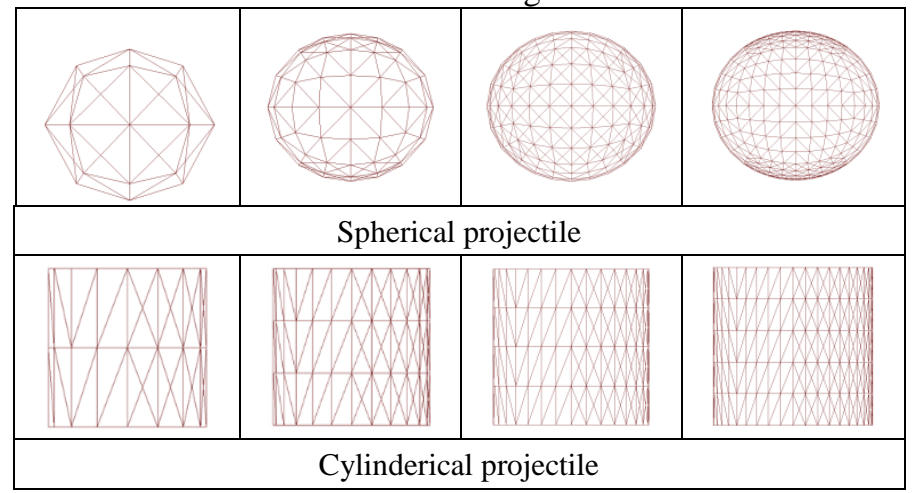

Figure 4. FE projectile with varies mesh density

Table 3 summarizes the simulation results of $\mathrm{FE}$ meshed spherical projectile using the modified DEA approach. The total nodes of FE meshed projectiles ranged from 53 to 2248 with a corresponding total element varying from 32 to 2037. The numerical maximum projectile force and bounce back velocity are compared to that of the previous DEA, and the discrepancies are listed below.

Table 3. Simulation Results for FE Spherical Projectiles

\begin{tabular}{|l|ccccc|} 
Test \# & 1 & 2 & 3 & 4 & 5 \\
Simulation time & 13 & 88 & 234 & 318 & 925 \\
(min) & 32 & 256 & 649 & 864 & 2037 \\
\# of elements & 53 & 321 & 779 & 997 & 2248 \\
\# of nodes & & & & & \\
Max. projectile & 793.5 & 950.1 & 992.1 & 990.4 & 994.5 \\
force (N) & -22.5 & -7.2 & -3.1 & -3.3 & -2.9 \\
$\begin{array}{l}\text { Discrepancy (\%) } \\
\text { Rebound speed } \\
(\mathrm{m} / \mathrm{s}) \text { 37.3 }\end{array}$ & 38.5 & 38.4 & 38.6 & 38.0 \\
Discrepancy (\%) & -3.0 & 0.28 & -0.028 & 0.34 & -1.3
\end{tabular}

Results of the maximum projectile force shows that, for spherical projectile, when the total number of nodes increases, the modified DEA simulation results more closely resembled the previous DEA results. However, when the total number of nodes reaches 779 or more, the simulation results between the modified DEA and the previous DEA approach are almost identical. The deviation of the bounce back speed is much smaller compares to the results of maximum projectile force. Approximately $2.7 \%$ improvement is noted between Test 1 and Test 2 on rebound speed. However, as the total number of nodes exceeded 779 , the discrepancy between the rebound speeds derive from the modified DEA and the previous DEA approach became bigger.

Table 4 summarizes the simulation results of FE meshed cylindrical projectile using the modified DEA approach. Similarly, simulated maximum projectile force and rebound speed are compared to results obtained from the previous DEA.

Table 4. Simulation Results for FE Cylindrical Projectiles

\begin{tabular}{|l|cccc|} 
Test \# & 1 & 2 & 3 & 4 \\
Simulation time & 35 & 115 & 296 & 667 \\
(min) & & 324 & 768 & 1500 \\
\# of elements & 96 & 484 & 1045 & 1926 \\
\# of nodes & 171 & & \\
Max. projectile & 345.6 & 354.0 & 356.1 & 354.0 \\
force(N) & -1.0 & -1.6 & -1.0 & -1.6
\end{tabular}




\begin{tabular}{|l|rrrr|}
$\begin{array}{l}\text { Rebound speed } \\
(\mathrm{m} / \mathrm{s})\end{array}$ & 34.6 & 35.2 & 35.1 & 34.9 \\
Discrepancy(\%) & -1.0 & 0.67 & 0.29 & -0.20
\end{tabular}

Likewise, similar conclusions can be reached for cylindrical projectile test results. Table 4 shows that discrepancies of both maximum projectile force and rebound speed decrease when the number of elements in projectile increases. The variation of the number of elements has a smaller effect on predicting rebound speed than it does on predicting maximum projectile force. However, the mesh density of cylindrical projectiles has significantly less effect on impact resistant performance than spherical projectiles. Less than $0.8 \%$ improvement on rebound speed is observed between Test 1 and Test 4 .

Figure 5 shows the simulation time steps vs. projectile force curve for both projectile type. The red curve is derived from the previous DEA; other curves are derived from the modified DEA approach. Discrepancies between the modified DEA and the previous DEA approach were visible in Test 1 . As mesh density increased, the simulation results of Test 3 to 4 and 5 closely match results from the previous DEA.

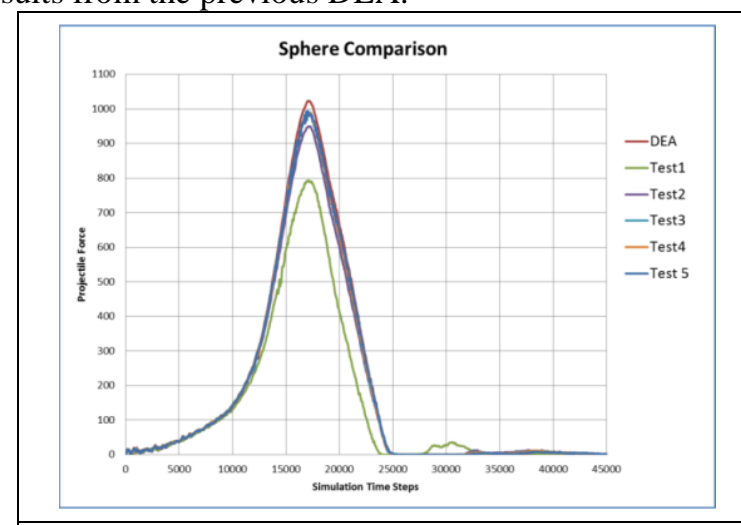

(a) Spherical projectile

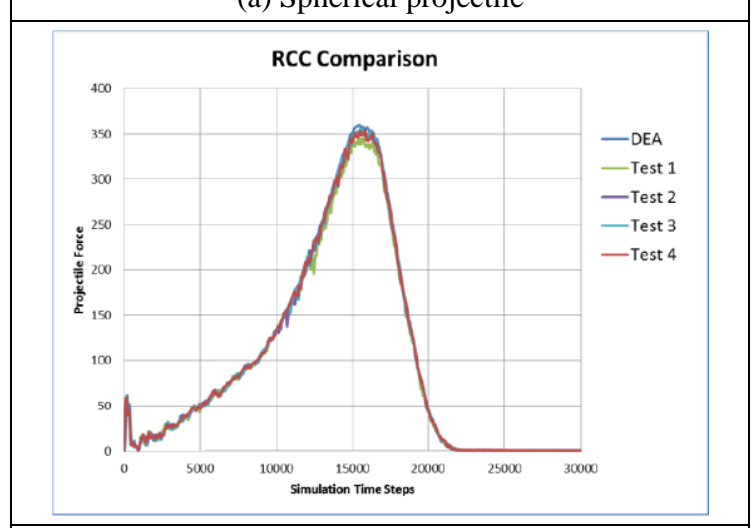

(b) RCC projectile

Figure 5. Projectile force comparison

\subsection{Conclusion}

Following conclusions are reached based on the above two sets of simulation:

1. Simulation results of the modified DEA approach using spherical and cylindrical projectiles closely resemble the results of the previous DEA. The modified DEA approach successfully replicates the results from the previous DEA and is capable of simulating ballistic impact of textile fabrics using projectiles of arbitrary shape. The numerical maximum projectile force and bounce back speed with respect to time curves match closely that of the previous DEA.

2. General speaking, the increase of mesh density improves simulation results. However, for standard spherical and cylinderical projectile, when the projectile total number of nodes reached 1000, the improvement of mesh density on simulation results became trivial, occasionally leads to slightly less accurate results than projectiles with coarser mesh.

3. Mesh density has a greater effect on spherical projectile than cylindrical projectile.

4. Because higher mesh density consumes significantly more computational time, a total number of nodes between 500 and 1000 is recommended for timeefficient simulation.

5. The modified DEA approach analyzer provides guidance for real bullet shape impact tests simulation.

\section{Acknowledgements}

This research is supported by the National Natural Science Foundation of China under Grant no. 51705058, the Science and Technology Research Program of Chongqing Municipal Education Commission under Grant no. KJ1704087, the Chongqing Research Program of Basic Research and Frontier Technology under Grant no. cstc2017jcyjAX0005, Chongqing Research Program of Basic Research and Frontier Technology (cstc2016jcyjA0594, cstc2016jcyjA0443), and the Scientific and Technological Research Program of Chongqing Municipal Education Commission (NO. KJ1600446, KJ1600422).

\section{References}

1. Chu Y, Min S, Chen X. Numerical study of inter-yarn friction on the failure of fabrics upon ballistic impacts. Mater. Des., 115:299-316 (2017)

2. Fang H, Gutowski M, Disogra M, et al. A numerical and experimental study of woven fabric material under ballistic impacts. Adv. in Eng. Software, 96:14-28 (2016)

3. Wang Y, Chen X, Young R, et al. A numerical and experimental analysis of the influence of crimp on ballistic impact response of woven fabrics. Compos. Struct., 140:44-52 (2016)

4. Ren C, Liu T, Siddique A, et al. High-speed visualizing and mesoscale modeling for deformation and damage of 3D angle-interlock woven composites subjected to transverse impacts. Int. J. of Mechanical Sci.(In Press)

5. Wang Y, Miao Y, Swenson D, et al. Digital element approach for simulating impact and penetration of textiles. Int. J. Impact Eng., 37(5):552-560 (2010)

6. Huang L, Wang Y, Miao Y, et al. Dynamic relaxation approach with periodic boundary conditions in determining the 3-D woven textile micro-geometry. Compos. Struct., 106:417-425 (2013) 
7. Ma Y, Huang LJ, Wang YQ, et al. Validation of explicit digital element dynamic relaxation approach in determining micro-geometry of 3-D woven fabrics. 27th Annu. Tech. Conf. of the Am. Soc. for Compos. 2012, Held Jointly with 15th Joint US-Japan Conf. on Compos. Mater. and ASTM-D30 Meeting, 1:235-246 (2012)

8. DFMA. http://www.fabricmechanics.com/. Kansas State University (2018)

9. Wang Y, Miao Y, Huang L, et al. Effect of the interfiber friction on fiber damage propagation and ballistic limit of 2-D woven fabrics under a fully confined boundary condition. Inter. J. of Impact Eng., 96:66-78 (2016) 